\title{
¿Es posible una razón crítica sin recurso a Ideas Regulativas? * \\ El nexo entre las dimensiones reflexiva y existencial de la crítica de patologías
}

\author{
LUIS SÁEZ RUEDA \\ Universidad de Granada
}

«Por la testimonial enseñanza de Amador Sáez Rueda»

\begin{abstract}
Resumen. Este trabajo se interroga por criterios normativos para una «crítica de patologías» en el ámbito de la cultura. La doctrina predominante al respecto en el siglo $\mathrm{xx}$ es la que J. Habermas y K.-O. Apel defienden, según la cual lo «patológico» se define como una distorsión de la racionalidad discursiva, cuyas condiciones ideales se consideran «idea regulativa» para la crítica. El autor sostiene, en primer lugar, que las distorsiones de esta «racionalidad» pueden ser desenmascaradas por medio de una «crítica negativa», para la cual no es necesario el recurso a ideas regulativas. En segundo lugar, mantiene que la «razón» del discurso no sería tal sin el subsuelo de una existencia pre-reflexiva y que entre una y otra existe una tensión insuperable, de carácter trágico, que es clave de la acción social. Finalmente, intenta dar cobertura normativa a una crítica existencial (y negativa) de patologias, que constituiría el opuesto necesario de la crítica ilustrada.
\end{abstract}

AbSTRACT. This essay deals with the normative criteria for a «critique of pathologies» within the scope of culture. In the $20^{\text {th }}$ Century, the dominant doctrine has been the one defended by J. Habermas and K.-O. Apel, according to which «pathologicals is defined as a distortion of discursive rationality, the ideal conditions for which are taken to be the «regulative idea» for the critique. The author maintains, first, that the distortions of such a «rationality» can be unmasked by means of a «negative critique», which does not demand resorting to regulative ideas. Second, it is argued that discourse's «reason» would not be such without a subsoil of pre-reflective existence and that there is a tragic and insuperable tension between both, where such a tension is central to understand social action. Finally, the author will attempt to offer a normative framework for the existential (and negative) critique of pathologies which be opposed to the enlightened critique.

* Este trabajo responde a la ponencia presentada en el Symposio Pragmatismus -Ohne regulative Ideen?, que tuvo lugar en Essen (Alemania) durante los dias 13 y 14 de junio de 1997, organizado por el Kulturwissenschaftliches Institut del Wissenschaftszentrum Nordrhein-Westfalen, con ocasión del setenta y cinco cumpleaños de K.-O. Apel. 
La cuestión fundamental que este trabajo se propone abordar, contenida en el título, invita a reflexionar sobre el vínculo entre los conceptos de «Pragmatismo» e «Idea Regulativa». Quisiera referirme, en primer lugar, a este lazo, con el fin de anticipar las cuestiones concretas que serán objeto de análisis y las opiniones que, al respecto, mantengo. Aunque la variedad de las concepciones pragmatistas contemporáneas es muy amplia, un rasgo común a todas ellas consiste en el descubrimiento del valor constituyente que posee la dimensión pragmática del lenguaje y de la acción respecto a los criterios de justificación racional e, incluso, respecto a lo que deba ser denominado «razón». Y en esta tesitura, el pragmatismo ha posibilitado dos perspectivas enfrentadas, en función de que se consideren las pretensiones universalistas de la razón como compatibles o no con la contingencia de la praxis. En todo caso, la idea de progreso surge siempre como problema cuando el pragmatismo entra en escena. La frontera entre las dos posiciones mencionadas podría ser trazada tomando, por ejemplo, como clave de análisis al pragmatismo americano. El pragmatismo americano había incluido desde el inicio una vinculación entre la experiencia y la apertura al futuro, pues entendía que nuestras creencias implican hábitos de acción y expectativas de comportamiento. Mediante su concepto «hermenéutico-trascendental» del lenguaje ${ }^{1}$, Apel ha ampliado el significado de la «máxima pragmática» peirceana ${ }^{2}$, mostrando brillantemente cómo en el proceso histórico del diálogo asistimos a una interpretación comunicativa del sentido lingüístico, y, a través de ello, del sentido del ser de las cosas. En este caso, la dimensión de futuro de la praxis comunicativa se descubre orientable por la Idea Regulativa y contrafáctica de una comunidad ideal de comunicación ${ }^{3}$. R. Rorty (partiendo de Dewey) vincula, por el contrario, la apertura al futuro, no a Ideas Regulativas, sino a criterios contingentes, pues entiende que el léxico de las afirmaciones sobre el mundo se teje en función de su pertinencia en la consecución de ciertos propósitos del agente, de metas que arraigan en prácticas sociales variables ${ }^{4}$.

Este contraste permite formular con alguna precisión la tesis más general que quisiera intentar defender a través de las subsiguientes reflexiones, a saber, que, contra el pragmatismo contextualista, es posible discernir criterios universales para la crítica del conocimiento y del comportamiento moral, pero que ello implica una modificación sustancial de lo que entiende Apel por progreso y por Idea Regulativa. De acuerdo con dicha modificación, los criterios universales de la crítica deben ser entendidos como condiciones negativas del ejercicio racional, es decir, como condiciones que indican qué no es racional, pero que no alcanzan a determinar positivamente lo racional. A esta conclusión fuerza, a mi juicio, la necesidad de hacer justicia a la dimensión prerreflexiva del Logos - del ser-racional como praxis o ejercicio-y su peculiar imbricación con la faz reflexiva y discursiva de la racionalidad. $\mathrm{El}$ autor retoma así su propuesta, formulada ya en otro lugar ${ }^{5}$, de entender el ejercicio de la racionalidad como puesta en obra de una tensión, al mismo tiempo insoslayable e insoluble, entre la "posición céntrica» del sujeto (ligada a la existencia prerreflexiva) y su "posición ex-céntrica» (reflexiva), proyectándola sobre el problema de la crítica racional.

El despliegue del referido horizonte general del trabajo atravesará los siguientes hitos. En primer lugar ( $\$ 1)$, intentará mostrar las debilidades de dos concepciones opuestas de la crítica: una que presupone la deseabilidad de un «cielo de la máxima pluralidad» (neopragmatismo americano); otra que confía, tácitamente, en un «ideal de convergencia» (pragmática trascendental). A continuación, intentará especificar, más allá de la pragmática tras- 
cendental, una tensión entre centricidad y ex-centricidad en los ámbitos de la autonomía, de la verdad, de la identidad y de la praxis moral (\$2). Finalmente, buscará definir el modo de crítica racional que es coherente con dicha disposición tensional del ser-racional ( $\$ 3$ ). La hipótesis por la que, en este último contexto, el trabajo apuesta, es la de que una crítica racional debe incluir un desenmascaramiento de patologías en dos órdenes del ejercicio de la racionalidad: en el reflexivo-discursivo y en el de la dimensión prerreflexiva de la praxis, caso, Este último, en el que se apela a una fenomenología crítica.

\section{EL ERROR DEL CONTEXTUALISMO Y LA INSUFICIENCIA DEL IDEAL DE CONVERGENCIA}

Frente al universalismo, ha defendido Rorty que todo impulso teórico está al servicio de la «creación de sí» $o$ al de la «perfección privada», y no al de la verdad o la solidaridad ${ }^{6}$. Para evitar el egocentrismo ético y el sufrimiento, cree Rorty que basta con aceptar la tradición política liberal, permitiendo a todos la posibilidad de crearse a sí mismos según sus capacidades. Lo demás, a su juicio, vendrá por añadidura: si nos cuidamos de la «libertad política - dice el pragmatista-, la verdad y el bien se cuidarán de sí mismos» ${ }^{7}$. Esta convicción de Rorty se basa en una fe ingenua no exenta de componentes místicos, a saber, la fe en la bondad inherente al «laissez faire», como si una especie de misteriosa «armonía preestablecida» garantizara la unidad entre la proliferación libre de lo heterogéneo y la consecución de la justicia y de una existencia rica. No otra cosa parece sugerir su invocación a una sociedad utópica en la que cualquier perspectiva posible sería bienvenida ${ }^{8}$. La percepción de la ingenuidad que comporta esta esperanza en un «cielo de la máxima pluralidad», basta para exigir, frente a Rorty, el despejamiento de una razón crítica capaz de poner freno al fenómeno que él obvia, a saber, el de las deformaciones siempre acechantes del diálogo y de la realización de sí.

Una de las mayores aportaciones de Habermas y Apel a la filosofía contemporánea consiste en la concepción de una racionalidad «centrada», que reconoce la doble vinculación de nuestra existencia lingüística a la facticidad de los contextos y, simultáneamente, a la idealidad de una situación de habla que es anticipada en virtud de las pretensiones de validez universal inherentes al diálogo. Esta paradójica estructura de la comprensión humana del mundo, que impele - como ha destacado Habermas - a «trascender desde dentro" todo provincialismo o contextualismo fáctico ${ }^{9}$, toma asiento, me parece, en esa doble «posicionalidad», «céntrica» (es decir, comprometida en el mundo) y ex-céntrica (es decir, capaz de autodistanciamiento) que Apel ha destacado con gran lucidez como constitutiva del pensamiento humano ${ }^{10}$. La posición excéntrica impele a un auto-rebasamiento hacia un espacio de universalidad irrestricta, lo que muestra -como dice Apel- «que el hombre está obligado, necesariamente, a anticiparse a sí mismo para ser hombre» ${ }^{11}$.

No creo que esta descripción de la textura de la racionalidad pueda ser negada sin incurrir en contradicción performativa. Ahora bien, me parece que la concepción apeliano-habermasiana ha interpretado esta tensión entre posición céntrica y excéntrica, entre facticidad e idealidad, de un modo incorrecto, al hacer coincidente el hipotético cumplimiento de las condiciones formales de un diálogo ideal anticipado con resultados que poseen un carácter material. Ofreceré dos ejemplos especialmente relevantes. El primero de ellos se refiere a una coincidencia entre el hipotético cumplimiento de tales condiciones formales y el logro - según 
Apel-de una síntesis de la interpretación, que es simultánea con una síntesis de la validación ${ }^{12}$. El segundo ejemplo se refiere al logro de un entendimiento sin fisuras entre «formas de vida». Pues icómo podrían acceder los miembros de una comunidad a un acuerdo completo si no es sobre la base de una forma de vida común que permitiese la «comprensión del sentido" de las pretensiones y puntos de vista de todos los participantes? El mismo Habermas ha reconocido esta copertenencia entre «situación ideal de habla» y la idea de una reconciliación, de una Versöhnung ${ }^{13}$.

Estos ejemplos muestran que la pragmática habermasiano-apeliana comprende la dimensión del futuro de un modo inverso al pragmatismo contextualista: no como un «cielo de la máxima pluralidad», sino como un «cielo de máxima convergencia», ciertamente inalcanzable, pero sí deseable y regulador.

Desde mi punto de vista, este espectro de coincidencias entre cumplimiento de condiciones formales ideales y logros de una convergencia en un sentido material es una mera suposición que no puede ser fundamentada pragmático-trascendentalmente. Resulta convincente que realizando una «autorreflexión estricta» acerca de nuestros actos de argumentación nos vemos obligados a reconocer que atribuimos la dignidad de enjuiciar la validez de nuestros asertos a un auditorio ilimitado. en condiciones de completa simetría. Pero cualquier otra consideración adicional introduce elementos «teóricos» que no pueden emerger simplemente de dicha «autorreflexión estricta» ${ }^{14}$. El espectro de convergencias mencionado supone, por ejemplo, que las multiplicidad de las interpretaciones es susceptible de ser subsumida en nexos de sentido más globales y univocos, que todo lo comprensible es potencialmente explicable mediante argumentos, que las fuentes de la heteronomía son sólo las de la comunicación distorsionada, o que la heterogeneidad de las formas de vida es potencialmente superable.

Por ello, yo no encuentro razones justificables a priori que me impidan imaginar lo contrario. Es pensable, sin autocontradicción performativa, que una imaginaria sociedad cumple escrupulosamente las condiciones formales de un diálogo sin coacciones y que, sin embargo, no accede a un entendimiento, o que accede a un acuerdo que versa sobre una verdad fútil e insignificante. Es pensable, también, sin contradicción una sociedad democratizada en la que fuesen seguidas escrupulosamente las condiciones de respeto y reconocimiento intersubjetivo y que, sin embargo, constituyese una sociedad cuya forma de autocomprensión fomentase identidades dependientes, incapaces de autonomía y un proyecto de existencia improductivo.

$Y$ es que la coincidencia entre cumplimiento de procedimientos formales $y$ resultados materiales genuinos implica la introducción de condiciones no formalizables. Acertadamente, ha mostrado Wellmer, por ejemplo, que la idea de un acuerdo verdadero presupone el cumplimiento de la «capacidad suficiente de juicio» de todos los participantes ${ }^{15}$. En esta línea, señala Kettner que «para que los consensos sean racionales, los participantes, no sólo han de poder hacer uso de su libertad comunicativa y de su capacidad de decisión, sino, además, de hacer un buen uso de ellas» ${ }^{16}$. Pero este «plus» arraiga, a mi juicio, en una dimensión prerreflexiva que es inherente a la razón. Dedicaré el resto de consideraciones a poner a prueba esta hipótesis.

\section{RACIONALIDAD DISCURSIVA E INTELIGENCIA PRERREFLEXIVA}

Procuraré mostrar la presencia de la dimensión prerreflexiva en cuatro ámbitos: en el de la autonomía, en el de la identidad, 
en el de la verdad y en el de la praxis ética. Y quisiera resumir antes la tesis que intentaré probar a propósito de cada uno de ellos: la acción racional incluye dos dimensiones, una reflexiva, vinculada a la posicionalidad excéntrica del sujeto, y otra prerreflexiva, que pertenece a su posicionalidad centrada. Están entrelazadas, pero entre ellas existe un hiato y una tensión insuperable en una superación (Aufhebung), ni siquiera bajo la forma de un ideal contrafáctico.

\subsection{Autonomia: tensión entre «autodeterminación» $y$ «mantenerse en sú}

Comenzaré rastreando esta tensión en el primero de los objetos de análisis que he mencionado. Utilizo aquí el concepto de autonomía, no en un estricto sentido moral, sino en el sentido amplio que introdujo Kant en su emocionante llamada a seguir el conatus ilustrado, a saber, como la capacidad para servirse con coraje y con valor del propio entendimiento sin la guía de otro. Esta misma invocación está contenida en la racionalidad discursiva. La autonomía implica en este contexto la capacidad excéntrica de distanciamiento para someter los contenidos materiales del mundo de la vida a la prueba de la reflexión dialógica ${ }^{17}$ y la capacidad para actuar de acuerdo con razones. Kettner ha subrayado, con razón, a mi juicio, que esta racionalidad autónoma es una forma de praxis orientadora (Orientierungspraxis) asistida por la conciencia. Pues bien, llamémosle a esta forma de autonomía y Orientierungspraxis «autodeterminación racional».

Llamo la atención ahora sobre el conocido y clásico caso de patología que $\mathrm{M}$. M.-Ponty comenta en la Phénoménologie de la perception. El enfermo Schneider es un hombre maduro y reflexivo, capaz de argumentar con consistencia, pero incapaz de situarse en contextos de sentido en el mundo de la vida y de dirigir su existencia. Y por ello se da la paradoja de que toda acción para Schneider tiene que obedecer para él a una razón, pero no encuentra «razones» en la experiencia inmediata que orienten de un modo situado su vida 18 Fenómenos semejantes comenta Blankenburg refiriéndose a esquizofrénicos ebefrénicos ${ }^{19}$. Algunos de estos enfermos están condenados a construir motivos racionales para la acción. No encuentran en la experiencia inmediata nexos de sentido que orienten su acción, por lo que se ven obligados a sustituir esa dirección comprensible de la que carecen por cadenas de razones. En ambos casos, estos pacientes están condenados a apoyar su acción en razones asistidas por la conciencia, pero no son autónomos, porque son incapaces de establecer un nexo entre reflexión y mundo de la vida capaz de permitir el «comprenderse sobre algo» (sich verstehen auf etwas) en el mundo. Carecen, por ello, de una «dirección» en la vida sensible.

Estos ejemplos podrían ilustrar la idea de que el valor que es perceptible en las razones a la luz de la conciencia no posee una fuerza orientadora suficiente para la acción. Para que orienten, deben poder mantener un vínculo con horizontes prerreflexivos cuya ausencia deja huérfanas a las razones, como si fuesen jugadas posibles sin un campo de juego ${ }^{20}$.

Por ello, creo que es necesario incluir en el concepto de autonomía una dimensión que afecta a la posicionalidad céntrica del sujeto. P. Ricoeur se ha referido a ella con un término con resonancias heideggerianas: la Selbst-ständigkeit ${ }^{21}$. En el sentido en el que a mí me gustaría utilizar este concepto, la «Selbst-ständigkeit» implica una capacidad para mantenerse en sí en la red de contextos del mundo de la vida, un poder para «atenerse a sí mismo» con fidelidad y valentía. Y ello sería inexplicable sin presuponer esa paradójica «pasividad activa» de la vida prerreflexiva que M. M.-Ponty llamó être brut (ser sal- 
vaje). Una pasividad, por cuanto significa un poder «permitir y permitirme ser», un "poder-dejarse-afectar» por las expectativas que la situación reclama y solicita desde sí, de tal forma que dicha solicitación penetre en la propia autocomprensión del sujeto e instale en ella demandas a las que éste ha de hacer frente "manteniéndose en sí» y «a través de ellas». Pero esta pasividad no constituye un «nihil negativum». Como dijo Merleau-Ponty, somos libres, no pese a nuestra comprensión centrada en contextos, sino gracias y a través de ella. Esa pasividad hace emerger una «actividad prospectiva» mediante la cual el sujeto se ve requerido a acciones y trayectorias posibles y responde organizando sin regla el espectáculo del mundo ${ }^{22}$.

Pues bien, estas dos dimensiones de la autonomía están interpenetradas. La «autodeterminación racional» quedaría huérfana $\sin$ la asistencia de esta orientación emergente. Pero, a su vez, esta última necesita el auxilio de la primera. Sin embargo, ambas son irreductibles la una a la otra. Ninguna configuración de argumentos puede sustituir a esta responsabilidad del «mantenerse en sí» ni puede traducir su riqueza significativa, pues las posibilidades de apelación que la experiencia situada contiene no son previsibles ni agotables en explicaciones discursivas. $\mathrm{Y}$, al mismo tiempo, las razones incluyen una pretensión de validez que trasciende todo contexto. Ambas dimensiones se relacionan, pues, en la forma de una tensión insoluble no susceptible de ser disuelta en una Aufhebung. Ser autónomo presupone el esfuerzo por mantener viva la tensión entre «autodeterminación racional» y «mantenimiento de sí», de forma que el polemos entre uno y otro se muestre productivo y ofrezca la medida de una «autonomía concreta».

\subsection{Sí mismo: tensión \\ entre «identidad discursiva» $e$ «identidad narrativa»}

Una figura semejante de pensamiento ayuda a entender el logro de la identidad. Como han mostrado Habermas y Apel, la constitución del Selbst es impensable sin presuponer en el sujeto un movimiento -en mi terminología, excéntrico - de vuelta a sí a través del reconocimiento de sus pretensiones por parte del alter ego. Ello exige que el sujeto sea capaz de distanciarse de sí y adoptar la perspectiva de la tercera persona, para comprender sus propias intenciones de sentido. De este modo, la constitución del Selbst implica, a la postre, la participación en los procesos de coordinación de la acción a través del reconocimiento recíproco de pretensiones de valide ${ }^{23}$. Llamémosle a esta dimensión de la identidad «identidad excéntrico-discursiva». Pues bien, a mi juicio, sería erróneo considerar a dicha dimensión excéntrica, no sólo como condición necesaria, sino como fuente generadora de la posesión de un «yo" propio. El mismo Habermas habla - a mi juicio, equivocadamente- del sí mismo (Selbst) como de una «autocomprensión resultante» («resultierende Selbstverhältnis») originada en la comprensión que el hablante logra de sí desde la perspectiva del otro ${ }^{24}$. Este proceso excéntrico no explica por sí mismo la aprehensión de la propia identidad como «inalienablemente mía». En este contexto, yo defendería, con P. Ricoeur, que el Selbst se forja, además, en el tejido de una identidad narrativa ${ }^{25}$. Ésta incluye el poder de comprender retroactivamente el polimorfismo de los acontecimientos vividos como una trama que posee nexos internamente comprensibles, y el poder prospectivo para proyectar nuevas posibilidades de autocomprensión. Que tales fuerzas - que delatan la presencia silenciosa de un «deseo de ser»-, no puedan adquirir forma sin proyectarse a sí mismas 
desde el espejo que representa el reconocimiento del otro, no significa que emerjan de esta retroproyección. Hemos de presuponer también el movimiento inverso como condición de la apertura al alter ego. ¿Desde dónde podría aprehender el sujeto el sentido del acto de reconocimiento del otro si no es desde una trama propia de anhelos, vicisitudes o valoraciones?

En este sentido, hay que comprender las tesis que hoy defiende Honneth como expresión de una verdad parcial. Sin reconocimiento -en ello lleva razón- no germinará la integridad y la identidad ${ }^{26}$. Pero el reconocimiento por sí mismo no garantiza una identidad genuina o una autorrealización comprometida y valiente. La dinámica de las sociedades no está trabada sólo por la fuerza negativa o de resistencia que impulsa a evitar el desprecio (Mißßachtung), sino también por la fuerza positiva de una responsabilidad insoslayable: la de hacerse a sí mismo con coraje y valentía. A veces, dicho sea de paso, esta valentía exige persistir en los propios proyectos incluso en el caso de que no obtengan reconocimiento. Y aceptar la imprevisibilidad del reconocimiento e, incluso, la posibilidad de fracaso, es un deber para quien quiera iniciarse a sí mismo.

Estas reflexiones fuerzan a admitir, a mi juicio, que entre «identidad excéntrico-discursiva» e «identidad céntrico-narrativa» existe una mutua presuposición e imbricación ${ }^{27}$. Es más, si el sujeto no fuese capaz de exponerse desde su biografía a la mirada del otro e intentar justificar las posibles ofertas que de ella emanan como si fuesen el producto de razones argumentables, ya no podría decir tampoco de su autocomprensión que es suya, pues -como afitma Tugendhat contra Heideggerde una autoelección que no pueda apoyarse en razones no puede decirse que sea una autoelección, sino sólo un suceso arbitrario ${ }^{28}$. Ahora bien, ambas dimensiones se relacionan en la forma de una tensión insuperable. Pues una imaginable Aufhe- bung significaría, no sólo que la trama que articula la propia identidad irrepetible necesita del reconocimiento y ha de ser capaz de apoyarse en razones, sino también que sería deducible a partir de este reconocimiento y de estas razones argumentables. $\mathrm{Y}$ entonces ya no podríamos explicar el vínculo entre justificabilidad y autoelección, entre los frutos de la necesidad racional y los dones del azar, o nos veríamos obligados a admitir que la racionalidad discursiva busca en su esfuerzo validatorio un secreto algoritmo que nos libraría, al fin, del esfuerzo por ser ${ }^{29}$.

\subsection{Verdad: tensión entre «constitución} del sentido» $y$ «justificación de la validez»

De nuevo me parece que una figura semejante de pensamiento podría iluminar también el problema de la verdad. Los dos polos en oposición en este ámbito han sido analizados con gran penetración por Apel. Se trata de las dimensiones de la «constitución del sentido» (Sinnkonstitution) a través del compromiso práctico en el mundo, por un lado, y de la «justificación de la validez» (Geltungsrechtfertigung), a través de la aportación de razones en el espacio del discurso argumentativo, por otro ${ }^{30}$. Ahora bien, presuponer, con Apel, un Ideal Regulativo que constituiría una síntesis de la interpretación y de la validación, implica que la "constitución del sentido" es susceptible de una justificación progresiva. El principio de «expresabilidad» de Searle se ha convertido aquí en lo que yo llamaría un «principio de justificabilidad discursiva» según el cual toda experiencia prerreflexiva del mundo es susceptible, en principio, de ser traducida sin merma en una configuración reflexiva de argumentos. Baste señalar que para Apel todas las acciones con sentido son ya argumentos virtuales ${ }^{31}$. Y ello provoca una segregación muy sutil de la experiencia prerreflexiva del espacio de la racionalidad discursiva: 
el «pre» de la «prerreflexión» es comprendido como indicativo de una condición carencial. Y cuando a las fuentes de la experiencia prerreflexiva se les permite un espacio en el cosmos del discurso es a condición de someterlas a la lógica de las pretensiones de validez, es decir, a condición de domesticarlas. En el discurso serio, tales fuerzas - dice Habermas- «en modo alguno ausentes, están, por así decirlo, domesticadas y puestas al servicio de los especiales fines de la resolución de problemas» ${ }^{32}$. Me gustaría mostrar que la dimensión de la «constitución del sentido» incluye un dinamismo prerreflexivo que no es ni segregable ni domesticable.

Quisiera señalar, en primer lugar, que no entiendo por elementos de la constitución del sentido meramente «facticidades» objetivables. Así es como Rorty entiende la base a la que se someten todas las justificaciones: como prácticas que, en sus propios términos, representan «todos ya siempre abiertos» y descriptibles ${ }^{33}$. Y así es cómo, me parece, entendía Wittgenstein en Über Gewißtheit, las certezas que actúan como goznes de las razones que pueden ofrecerse en el seno de cualquier «Sprachspiel»: como convicciones fácticas que tienen su origen en la tradición, la educación o la persuasión y que no pueden ser justificadas, sino sólo descritas, constatadas. Si entendemos los elementos de la constitución del sentido de este modo resulta fácil justificar que la heterogeneidad o la pluralidad no está reñida con el consenso y la síntesis. Pues, como ha mostrado Apel, las certezas que articulan los juegos lingüísticos concretos son revisables en el seno más amplio del discurso mediante el ejercicio de la crítica, lo que permite comprender la posibilidad de un falibilismo en consonancia con el «meliorismo» $y$, por tanto, con un proceso de convergencia paulatino ${ }^{34}$.

Entiendo por elementos responsables de la «constitución del sentido», más bien, «fuerzas» o "poderes» asociadas con «ca- pacidades», que pueden sedimentar en «facticidades descriptibles» (las prácticas sociales, según Rorty, o las certezas de todo juego lingüístico, según Wittgenstein, en Über Gewißthet), pero que en sí mismos son fenómenos o acontecimientos inobjetivables. Ciertamente, Apel no ha sucumbido, como Rorty, a una naturalización de los elementos de constitución del sentido, pues reconoce, por ejemplo, que los «razonamientos abductivos" van ligados a actos como la creación de hipótesis innovadoras o la percepción de nuevos sentidos de los fenómenos (Phänomene), pero está convencido de que tales maravillas son compatibles con la idea de una síntesis perfeccionable in the long run ${ }^{35}$. Y esto es lo que quisiera poner en cuestión.

Uno de los fenómenos a los que me gustaría referirme es el de la «posición interrogante». Cuando un argumentante afirma algo pretende validez, pero, al mismo tiempo, está respondiendo a la demanda de una posición interrogante ante el enigma del mundo. Sin el presupuesto de este posicionamiento, ninguna pretensión de validez sería comprensible como expresión de un reto en la existencia. Tal posicionamiento es un acontecimiento que Merleau-Ponty comprendió justamente como el de una interrogación informulable que está detrás de todas nuestras interrogaciones formulables, porque es un modo de situarse en el mundo ${ }^{36}$. Este fenómeno ha sido brillantemente aclarado por Waldenfels mediante su distinción entre «Warum» o «Was» Fragen - preguntas que surgen en el contexto de un discurso ya abierto y que aspiran a llenar una laguna- $\mathrm{y}$ «Worauf-Fragen», que constituyen una exhortación y que abren el campo de juego en el que se hacen posible las preguntas del primer tipo ${ }^{37}$. Pues bien, la posición interrogante no representa meramente una condición psicológica o motivacional que interviene en la génesis de los juicios. Genera un modo de experiencia, porque permite el dejarse afectar y la emergencia 
de expectativas y de horizontes, es decir, la «posesión de un mundo». Y, por ello, esta experiencia prerreflexiva no debe ser confundida con una mística captación de contenidos materiales $y$, por tanto, con una forma misteriosa de conocimiento que es potencialmente actualizable bajo una forma reflexiva. Más bien es un modo de organizar la experiencia posible y de convocar al juicio a afrontar un reto. Sería mejor comprenderla, pues, como una nervadura que queda conservada en el ejercicio de los juicios. Así, por ejemplo, a menos que entendamos de forma algorítmica el modo en que discurren los argumentos, hemos de reconocer que en la elección de afirmaciones pertinentes, en los nexos entre argumentos o en los ensayos que conforman un perfil reflexivo, ha sido ya anticipada de forma prelógica una trama de enlaces o una retícula de caminos.

Esto no quiere decir que la experiencia forjada argumentativamente sea reductible a la experiencia prerreflexiva. Las formas de argumentación poseen, al mismo tiempo, un poder propio en virtud de sus pretensiones de validez, lo que explica que se emancipen de la fuente que los requiere y se plieguen a la fuerza de la lógica discursiva, e incluso, que reviertan retroactivamente sobre la experiencia prerreflexiva y operen cambios en ella. Pero sería absurdo pensar que esta última es susceptible de ser traducida sin merma en una configuración de razones. Entre ambos ámbitos existe un hiato insuperable. En primer lugar, porque son heterogéneos. La materia que conforma la posición interrogante es la de la apelación, la del sentirse convocado, no la de las pretensiones de validez. Merleau-Ponty aludió a ello de modo emocionante cuando dijo que la relación entre la inmersión en la experiencia y las propiedades de las cosas que ésta revela es la que existe entre el «yo puedo» y las maravillas que éste es capaz de suscitar $^{38}$. En segundo lugar, porque no hay una mathesis de las formas de interrogar al mundo. La emergencia de éstas no es calculable, es imprevisible e indomeñable. Y ello implica también que las posibilidades de interrogación son inagotables, por lo que la riqueza de la experiencia prerreflexiva excederá siempre los esfuerzos excéntricos de justificación argumentativa.

La reflexión anterior muestra que los puntos de vista que se ponen en juego en el discurso colisionan simultáneamente en dos planos. Por un lado, en el plano «horizontal» de la justificación, del juego de afirmaciones, réplicas y contrarréplicas. Por otro, tiene lugar un encuentro entre formas «verticales» de afrontar el mundo. «Verticales», porque se trata, en este caso, del nexo que enlaza a cada punto de vista con sus fuentes prerreflexivas. Ambas están interpenetradas en el espacio del diálogo. Y si la dimensión horizontal aspira a un acuerdo, a una síntesis última, esta dimensionalidad «vertical» la impide por principio. No sólo, porque, como he señalado, las posibilidades que puede abrir son inagotables, sino porque cada una de ellas implica una acotación de la experiencia del mundo que, como ha señalado Waldenfels, lleva aparejada una simultánea exclusión y segregación de otras posibles, lo que introduce una fragmentación insuperable en la trama interna del discurso e impide un progreso asintótico o una síntesis total ${ }^{39}$.

$\mathrm{Si}$ mis afirmaciones acerca de esta dimensión vertical del encuentro intersubjetivo merecen crédito, estaríamos autorizados, por lo demás, a pensar el discurso, al mismo tiempo, como un espacio de intercorporeidad, un espacio de interafección que presupone una recíproca apelación a aprehender las significaciones silenciosas de la posición interrogante. Pero como una respuesta a esta demanda implica, tanto una apertura al otro, una desposesión, como una apropiación creativa del sentido por parte del que escucha, la universalidad de la recíproca apelación estará siempre 
fragmentada y siempre «en estado naciente» ${ }^{40}$. Supuesto esto, habría que señalar que la idea de una comunidad de comunicación argumentativa presupone, al mismo tiempo, la idea de una «comunidad de sentimiento» o de «interafección». Pero este doble componente del ideal de entendimiento contiene una exigencia autocontradictoria, la de traducir discursivamente la riqueza ingobernable de la interafección, la de presentar (Vor-stellen) lo que por principio excede toda presentación. Y esta reflexión podría confirmar la tesis de Lyotard, según la cual el ideal de una comunidad irrestricta constituye una expresión del sentimiento de lo sublime, que según Kant procede de la impotencia de la imaginación para presentar una idea ${ }^{41}$. Ello pone de manifiesto, a mi juicio, que la no realizabilidad del ideal no consiste meramente en que sea contrafáctico, sino, además, en que contiene demandas cuya compatibilidad es para nosotros impensable.

Otro factor inherente a la dimensión de la «constitución del sentido» que me gustaría mencionar es la intervención en éste de un momento de «indisponibilidad». Creo que este fenómeno ha sido excelentemente estudiado por Lyotard. Cualquier forma de discurso "eslabona» proposiciones en función de un fin. Ahora bien, no hay una necesidad trascendental o lógica que determine el «eslabonamiento». Otra infinidad de eslabonamientos posibles podrían haber ocupado su lugar. Y cada uno de ellos habría introducido una distribución de líneas de fuerza distinta, que es lo mismo que decir que hubiera hecho comparecer a «lo real» bajo un rostro distinto. Esto significa que la ocurrencia de los encadenamientos no viene determina-

- da rígidamente por las intenciones de los agentes. Más bien ocurre - como dice Lyotard- que las finalidades de cada discurso «inspiran» formas de eslabonamiento posibles entre los cuales habría siempre una «diferencia». Pero, además, la actualización de cada uno de ellos reduce nece- sariamente al silencio a otros posibles. Ninguna intencionalidad de sentido puede evitar este silencio simultáneo. Genera inintencionadamente una diferencia entre lo expresado y lo expresable que no puede gobernar y cuyos efectos escapan a la intención del agente ${ }^{42}$. Estos fenómenos introducen en el uso del lenguaje un espacio de indeterminabilidad y de inmanipulabilidad ${ }^{43}$. Me parece, sin embargo, que admitiendo este hecho, habría que entenderlo, frente a Lyotard, sólo como un elemento perteneciente a la posicionalidad céntrica, pero que no anula la excéntrica ${ }^{44}$.

Por todas las razones aducidas anteriormente, creo que es necesario concluir que la dimensión céntrica de la «constitución del sentido" y la dimensión excéntrica de la «justificación de la validez» están interpenetradas y son irreductibles la una a la otra. Se relacionan en la forma de una tensión insoluble en una Aufhebung.

\section{4. Ética: tensión entre «discursividad $y$ «promoción de existencia»}

También en el problema de la moral es posible discernir una figura semejante de pensamiento. Uno de los polos en juego en este ámbito es, sin duda, el que ha aclarado la ética discursiva. Me refiero a las condiciones formales de un diálogo sin coacciones y a la transformación que ha propiciado del principio de universalizabilidad kantiano. La argumentabilidad y el reconocimiento intersubjetivo son, en este caso, condición de la validez de las pretensiones con carácter normativo. Llamémosle a este aspecto de la acción moral «dimensión discursivo-universalizadora» de la praxis ética. Al otro polo en juego lo llamaré «promoción de existencia».

El término es de J. Nabert, a cuyos penetrantes análisis en el ámbito de la moral desearía hacer aquí honor ${ }^{45}$. Comenzaré haciendo alusión a un ejemplo del propio autor que nos introducirá en 
el sentido de este concepto. La transgresión de una norma moral puede dar lugar a una experiencia de culpa, y el incumplimiento de un ideal a una experiencia de fracaso. Pero estas experiencias no agotan su sentido en la relación entre la acción y la norma o el ideal, sino entre una acción y el campo entero de la existencia del sujeto. Si son genuinas, expresan el sentimiento de que nos hemos quedado más acá de nosotros mismos, que no hemos estado a la altura de una demanda secreta que ahora entendemos. Y fuerzan, en este caso, no a una mera reparación de nuestro comportamiento en este punto concreto que representa la transgresión de la norma o el incumplimiento del ideal, sino que impelen a ejercer una conmoción en la textura entera de nuestra actitud vital. El incumplimiento del deber es experimentado, no sólo como inadecuación entre acto y regla, sino como inadecuación entre lo que somos y lo que queremos ser. Por eso, para Nabert los deberes, si bien tienen un fundamento en sí mismos, representan un momento necesario de una aspiración más radical: el deseo de ser. Progresar moralmente implica no sólo progresar en el cumplimiento de deberes, sino profundizar este anhelo, es decir, "promoción de existencia» ${ }^{46}$.

Pues bien, creo que se puede extraer de la obra de Nabert un apoyo a mi hipótesis de que estas dos dimensiones, la «discursivo-universalizadora" y la "promoción de la existencia», se interpenetran y son irreductibles la una a la otra. Como ha tratado de mostrar Nabert, la «promoción de la existencia» necesita del ejercicio de la justificación de razones: sin ella ni siquiera podríamos saber lo que queremos. Al mismo tiempo, el ejercicio de la justificación es parte integrante del deseo de ser ${ }^{47}$. Ahora bien, entre ambos existe un hiato insuperable. En primer lugar, porque la promoción de la existencia implica extraer de sí fuerzas prerreflexivas que establezcan nuevos cauces para la acción $\mathrm{y}$, con ello, la percepción creativa de nuevos valores que no se deducen de una regla previa, sino que han de ser generados sin regla, en el magma versátil y oscuro de nuestra trayectoria existencial. Abstraídos de ese magma, los valores generados deben ser sometidos a la prueba de la crítica reflexiva. Pero cometeríamos un error si redujéramos su justificabilidad a los resultados de esta prueba. Una vez explicitadas, estas razones aparecen ficticiamente como causa de la acción. Las razones parecen fundarse unas en otras, con independencia de la dimensión de su génesis. Y si el sujeto cayese víctima de la tentación que supone esta abstracción, para atenerse simplemente a los resultados normativos de la reflexión crítica, perdería de vista la responsabilidad de afrontar los retos concretos en los que dichos valores habían emergido $^{48}$. Existe un hiato, en segundo lugar, porque la existencia está trabada por un conflicto real que queda diluido en el espacio del discurso. Así, por ejemplo, cuando afirmamos que una acción no es valiente afirmamos también que es cobarde. La cobardía, en la existencia, no es una falta de valentía, es un fuerza efectiva que tiene sus condiciones de generación y a la cual debe hacer frente el sujeto. Pero cuando se mide la acción en función de su ajuste o no a una norma ideal o al criterio de justificabilidad argumentativa, el contravalor es visto como «ausencia» del opuesto, como deficiencia ${ }^{49}$.

Desde mi punto de vista, esta dimensión que hemos llamado «promoción de existencia» exige el ejercicio de un juego de fuerzas prerreflexivas que no es reductible a la Phrónesis que se supone necesaria para la aplicación de una regla, pues demanda, como se ha dicho, la creación de valores, es decir, de horizontes para la acción sin regla previa. Y, por lo mismo, no es reductible a la «buena» voluntad que exige la acción «por mor" del deber. Tampoco representa meramente el elemento motivacional del que deben hacerse cargo los 
agentes. Es una responsabilidad, ni segregable de la dimensión discursiva - porque la dinamiza internamente-, ni domesticable por ella, porque - como he intentado mostrar - hay un hiato que las hace heterogéneas. Ambas dimensiones se relacionan, pues, en la forma de una tensión insuperable en una Aufhebung.

\section{LAS DOS DIMENSIONES DE UNA RAZÓN CRÍTICA}

A lo largo de estos cuatro temas de la exposición precedente he intentado mostrar que el ejercicio de la racionalidad comporta dos condiciones de posibilidad: una, ligada a nuestra posicionalidad céntrica y que remite a nuestro compromiso práctico-material con el mundo; otra, asociada a nuestra posicionalidad excéntrica, que nos fuerza a una autotrascendencia haciendo honor a ideales que trascienden todo contexto. He intentado mostrar que ambas dimensiones se interpenetran, pero que mantienen entre sí una tensión no susceptible de ser disuelta en una Aufhebung. Este punto de vista permite, a mi juicio, comprender que las fuerzas prerreflexivas que he indagado en el orden de la condición céntrica de la existencia no son ni segregables ni domesticables. No son segregables porque no constituyen un místico «más allá» de la racionalidad discursiva o un impensable «otro de la razón», pues penetran «desde dentro» la textura del Logos discursivo y son condición positiva de su vigor. No son domesticables, porque no son simplemente fuerzas parasitarias respecto a la dynamis de la resolución de pretensiones de validez. Pertenecen a una dimensión «vertical» del discurso cuyas demandas y cuya productividad no es reconstruible, traducible o sustituible en la dimensión «horizontal» de la justificación argumentativa.

El concepto de racionalidad apeliano-habermasiano tiene la virtud de haber iluminado a su modo esta doble dimensionalidad del Logos. Pero, como aludí al inicio, los filósofos de Frankfurt han comprendido erróneamente la relación entre ambos polos. El espectro de coincidencias ideales al que me referí al inicio entre cumplimiento de condiciones formales y logros materiales concretos fuerza a pensar el progreso como una ruptura paulatina de la tensión y el hiato entre los dos polos en juego en favor de un idealismo soterrado que somete las potencias de la facticidad al yugo de la idealidad. Ciertamente, la circunstancia de que este ámbito de convergencia final constituya una Idea Regulativa jamás realizable, implica que el polo de la facticidad no es superable por principio. Pero el modo en que esta insuperabilidad es pensada a la luz del ideal de convergencia, de transparencia y de justificación completas, presupone comprender los elementos de la dimensión prerreflexiva como límites y obstáculos de la racionalidad, y no como potencias positivas que penetran internamente a ésta y le confieren vigor y dignidad ${ }^{50}$. Empleando una metáfora: la dimensión prerreflexiva de la existencia es comprendida por Apel y Habermas como una sombra susceptible de ser mermada mediante un proceso inacabable de iluminación progresiva. En cambio, el punto de vista que he intentado mostrar sugiere otra metáfora distinta: la de que esta sombra puede ser desplazada del lugar que ocupa o transformada en cuanto al perfil que dibuja, pero nunca mermada. No es una dimensión medible a la que quepa restar cantidades. Es una magnitud cualitativa que acompaña oscuramente a la luz de la reflexión como el ojo acompaña a lo que ve sin verse a sí mismo.

Esta dimensión prerreflexiva no designa un lugar de ausencia, sino de presencia viva de demandas y aspiraciones. Ahora bien, el atenerse a estas demandas no cuenta con una idea regulativa. Ni el «mantenerse en sí», ni la «identidad narrativa», 
ni la «posición interrogante», ni la «promoción de existencia», poseen un ideal de perfectibilidad imaginable. Y, sin embargo, han de contar, respectivamente, como ingredientes ineludibles del horizonte de la autonomía, de la identidad, de la verdad y del comportamiento ético. Por ello, considero necesario renunciar a comprender las condiciones formales de la comunidad ideal de comunicación como una idea regulativa para el progreso, porque son condiciones necesarias de éste, pero no suficientes ${ }^{51}$

Es ahora cuando nuestra pregunta inicial adquiere mayor relevancia: ies posible, entonces, una crítica racional sin recurso a Ideas Regulativas? La respuesta, desde mi punto de vista, es positiva. Para justificarla invito a realizar una inflexión en la comprensión del lugar que ocupan las condiciones formales de un diálogo ideal. Éstas constituyen, en un sentido negativo, condiciones que indican qué formas de acción no son racionales, pero no, en un sentido positivo, condiciones que explicitan en qué consiste el genuino ejercicio de la racionalidad y regulan desde una lejanía ideal el proceso de acercamiento. Estoy de acuerdo, en este punto con Schnädelbach cuando afirma que la racionalidad es un sistema limitado por reglas determinables, y que, sin embargo, no puede ser expresado en su totalidad a través de ellas ${ }^{52}$. Y, en este sentido, me adhiero a la tesis de Wellmer según la cual el progreso puede ser entendido, no en la forma de un progresivo crecimiento en el ámbito del sentido, sino en la línea de una eliminación del sinsentido ${ }^{53}$.

Pero me gustaría añadir algo más. Es obvio que esta comprensión negativa del progreso aporta criterios, también negativos, de la crítica. Toda la teoría crítica habermasiano-apeliana se hace merecedora, desde este punto de vista, de un valor propio que ni el pragmatismo contextualista americano, ni la hermenéutica radical gadameriana, ni el pensamiento de la dife- rencia francés pueden desacreditar. Pues se trata, en este ámbito, del desenmascaramiento de patologías que distorsionan la posibilidad de un diálogo libre (en el sentido negativo que acabamos de sugerir).

Ahora bien, este ejercicio crítico está destinado a preservar las condiciones necesarias de la justificación de pretensiones de validez y de la evaluación argumentativa de nuestras concepciones, es decir, a preservar la salud de nuestra posicionalidad excéntrica. Conciernen a las condiciones de los polos que he llamado «autodeterminación racional», «identidad excéntrico-discursiva», «justificación de la validez» $\mathrm{y}$ «dimensión discursivo-universalizadora» de la praxis ética. ¿Es posible hablar también de patologías respecto a los polos respectivamente opuestos a los anteriores, que he vinculado a la posicionalidad céntrica, y en este sentido, de una crítica posible en este terreno? Aventuraré una respuesta afirmativa.

Las fuerzas prerreflexivas que operan a la sombra de la «luz racional» están expuestas continuamente al riesgo de desfallecer. Y este fenómeno autoriza a contradecir la tesis de Habermas según la cual las anomalías del diálogo deben ser entendidas sólo como «consecuencia de soluciones fallidas y de respuestas no válidas» y no también como «síntomas de un decaimiento de la vitalidad» ${ }^{54}$. Ofreceré algunos ejemplos de tales anomalías. En el ámbito de la autonomía, un desfallecimiento del poder para dar espacio a esa «pasividad activa» que es condición del «mantenimiento de sí», puede dar lugar a un individuo o una sociedad enferma que ya sólo aspira a una «libertad negativa» —una ausencia de trabas para la acción-, y no al ejercicio de una «libertad positiva», capaz de extraer, desde la escucha de lo que los contextos vitales reclaman desde sí, nuevas orientaciones para actuar con coraje y valentía.

Un desfallecimiento semejante en el ámbito de la «identidad narrativa» puede 
dar lugar a una sociedad enferma en la que los individuos sean perfectamente capaces de dirigir su vida en función de razones justificables, pero sean incapaces de inventar nuevas formas de autorrealización, nuevas tramas vitales. Puede dar lugar a una sociedad racionalizada en la que la necesidad de justificar todas las acciones erija a la autorreflexión en un juez implacable que mira con sospecha a los actos espontáneos y a la voluntad de aventura; o, tal vez, a una sociedad en la que el valor de la propia identidad se mida sólo por el factum del reconocimiento y no también por la anticipación del reconocimiento digno, mediante la percepción de formas de reconocimiento que ocultan una debilidad. Formulándolo de un modo que afecta a la tesis ya mencionada de Honneth según la cual la lucha por el reconocimiento (que él entiende - de modo negativocomo esfuerzo por evitar la Mißachtung) constituye el resorte que dinamiza el proceso social: sin presuponer una dimensión prerreflexiva que orienta positivamente a articular la propia biografía según estimaciones de grados de excelencia en los proyectos, icómo podríamos distinguir entre una aspiración - poco excelente- al reconocimiento (como la mera aspiración al prestigio profesional), de otra -más excelente- (como la aspiración al reconocimiento del propio esfuerzo?); ¿cómo podríamos distinguir una búsqueda de reconocimiento basada en la necesidad de suplir así la falta de una vida propia y rica, de otra basada en la necesidad de poner a prueba aquello por lo que apostamos y aquello en lo que «nos ponemos»? Tales estimaciones de grados de excelencia no surgen simplemente de la reflexión o del reconocimiento. Arraigan en una forma de autoafección prerreflexiva cuyo vigor puede crecer o decaer, mantenerse sano o enfermar.

En el ámbito de la verdad, un desfallecimiento, por ejemplo, de lo que he llamado «posición interrogante», puede dar lugar a una comunidad enferma capaz, quizás, de eliminar fuerzas extrañas que distorsionan la dimensión horizontal del diálogo argumentativo y que provocan autoextrañamiento (Selbstentfremdung), pero que ya no «se extraña» ante el enigma del mundo y es incapaz ya de esa disposición de escucha que permite la emergencia de retos nuevos para la inteligencia.

Finalmente, un desfallecimiento semejante en la dimensión de la «promoción de existencia» puede dar lugar a una sociedad enferma, judicializada, preocupada sólo por el imperativo de ajustarse a normas justificables, pero que haya perdido la facultad para percibir los conflictos reales de la existencia y la llamada a transformar, no sólo conductas concretas, sino, si es necesario, el horizonte entero bajo el cual discurre el deseo de ser y en el medium del cual valoramos el modo en que estamos o no a la altura de nosotros mismos.

Todas estas formas de patología - y otras innumerables que podríamos investigar - no son corregibles mediante una simple expansión de las posibilidades de participación en un discurso sin coacciones, tal y como la teoría de la racionalidad más expandida en la actualidad supone, sino que exigen, además, nuevas formas de hacer discurso. La salud, en este caso, concierne al ejercicio de una «razón sintiente» que arraiga - lo diré una vez másen ese vínculo entre inteligencia y naturaleza que representa el «ser salvaje» de la existencia y que constituye la infancia la razón, una infancia que ésta no supera sino que ha de conservar en su seno si pretende madurez. La salud, en este caso, concierne a una «razón ponderativa», capaz de percibir grados de excelencia; una razón «discriminativa», capaz de percibir relieves, distinciones en cada caso entre lo relevante y lo prescindible; una razón «apelativa», capaz de dejarse penetrar por el poder de la facticidad para invocar, para exhortar, y capaz, también, no sólo de cam- 
biar el orden horizontal del discurso, sino de apelar o conmover en el orden vertical.

Pues bien, ¿disponemos de criterios para la crítica en este ámbito? Desde luego, no en un sentido positivo. La pretensión de poner una medida a los grados de excelencia, de valentía, de autoafección, de pasividad productiva, etc., constituiría el ejercicio de un terror implacable, pues ¿quién podría erigirse en juez de semejante tribunal sin imponer sus propias medidas y pesos? Sin embargo, creo que podemos intentar investigar condiciones negativas, limitativas, que no miden positivamente grados de ascenso vital, de vigor o de compromiso, sino que indican condiciones sin las cuales no habría en absoluto ninguna posibilidad de ascenso, de vigor o de compromiso. Tales investigaciones deberían estar a cargo, a mi juicio, de una reflexión fenomenológica posthusserliana. En este sentido, me parecen aprovechables las producciones de autores como Jocelyn Benoist, J.-F. Courtine, Natalie Depraz, Didier Frank, Michel Haar o Dominique Janicaud, en el ámbito francés ${ }^{55}$, las de Waldenfels en Alemania y las de D. Blanco en España ${ }^{56}$. Este proyecto se presta también a una interdisciplinariedad entre filosofía y otras disciplinas, bien pertenecientes al ámbito de la psicología - como los análisis fenomenológico-psiquiátricos realizados por Blankenburg o las recientes investigaciones en torno a la «inteligencia emocional», entre las que cuentan las de D. Goleman ${ }^{57}$, bien al ámbito, incluso, de la neurología, tales como las que se están desarrollando en Norteamérica por autores como A. R. Damasio ${ }^{58}$.

El sentido de tales investigaciones posee también, a mi juicio, un potencial emancipador, pues todas estas formas de posible patología pueden ser interpretadas como una disminución del conatus o esfuerzo por existir, una experiencia cuyo reverso - como dice P. Ricoeur- es la disminución del poder de obrar y, consecuentemente, la generación de sufrimiento ${ }^{59}$. Sería tam- bién, una investigación de condiciones universales, aunque siempre falible. Universales, porque, como señalé al principio, de lo que se trata en este ámbito no es de facticidades culturales, sino de fuerzas o fenómenos. Tales fuerzas pueden ser expresadas en multitud de formas culturales o históricas, pero en sí mismas conforman un poder o capacidad, un Vermögen, sin el cual no sería posible la salud de ninguna cultura. No son universales, pues, en cuanto a las formas concretas en que sedimentan, por lo que se ajusta mejor a su sentido atribuirles los calificativos, no de «supra-culturales» o «supra-históricas», sino los de «trans-culturales» o «trans-históricas» ${ }^{60}$.

Quisiera añadir, finalmente, que si mis argumentos merecen crédito, sería lícito afirmar que un criterio normativo de tales investigaciones debería ser el mantenimiento de la tensión entre las dimensiones que hemos llamado sposicionalidad céntrica» $\mathrm{y}$ "posicionalidad excéntrica», pues el relajamiento de alguno de estos compromisos en favor de una invasión por parte del otro autoriza al investigador a sospechar que alguna patología se ha adueñado en ese caso del escenario de la acción racional.

\section{BIBLIOGRAFIA}

APEL, K.-O. (1973): Transformation der Philosophie, Frankfurt a. M., Suhrkamp. - (1976): «Das Problem der philosophischen Letztbegründung im Lichte einer transzendentalen Sprachpragmatik: Versuch einer Matakritik des "kritischen Rationalismus"», en KANITSCHEIDER, B. (ed.), Sprache und Erkenntnis, Innsbruck, pp. 55-82.

- (1987), «Fallibilismus, Konsenstheorie der Wahrheit und Letztbegründung», en Forum F. PHILOSOPHIE BAD HOMBURG (ed.), Philosophie und Begründung, Frankfurt a. M., Suhrkamp. 
- (1988), Diskurs und Verantwortung, Frankfurt a. M., Suhrkamp.

- (1989), «Sinnkonstitution und Geltungsrechtfertigung. Heidegger un das Problem der Transzendentalphilosophie», en Forum für PhilosophIE BAD Homburg (ed.), Martin Heidegger: Innen-und Aussenansichten, Frankfurt a. M., Suhrkamp, pp. 131-176.

- (1996): «Die Vernunftfunktion der kommunikativen Rationalität», en APEL, K.-O., y KETTNER, M. (ed.), Die eine Vernunft und die vielen Rationalitäten, Frankfurt a. M., Suhrkamp.

Blanco Fernández, D. (1989): «iUn delirio de la virtud?", en MuguerzA, J., y Rodríguez Aramayo, R., Kant después de Kant, Madrid, Tecnos, pp. 87-117.

- (1990): «Autonomía moral y autarquía», en GonZÁLez, J. M., y THIEBAuT, C. (eds.), Convicciones políticas y responsabilidades éticas, Anthropos, pp. 41-69.

- (1994): «Libertad», en CortinA, A. (ed.), Palabras claves en ética, Navarra, Estella pp. 203-289.

- (1998): «Descartes y el legado de la fenomenología», $E r$, núm. 23, juniojulio.

BlankenBuRG, W. (1971): Der Verlust der natürlichen Selbstverständlichkeit, Stuttgart, F. Enke Verlag.

FRANK, M. (1988): Die Grenzen der Verständigung -Ein Geistergespräch zwischen Lyotard und Habermas, Frankfurt a. M., Suhrkamp.

Goleman, D. (1996): Emotional Intelligen$c e$, Londres, Bloomsbury.

HAAR, M. (1994): Heidegger et l'essence de l'homme, Grenoble, Millon.

HABERMAS, J. (1981): Theorie des kommunikativen Handelns, Frankfurt a. M., Suhrkamp.

- (1986): Der philosophische Diskurs der Moderne, Frankfurt a. M., Suhrkamp.

- (1991a): Texte und Kontexte, Frankfurt a. M., Suhrkamp.

- (1991b): Erläuterungen zur Diskursethik, Frankfurt a. M., Suhrkamp.
- (1992): Nachmetaphysisches Denken, Frankfurt a. M., Suhrkamp.

HonNeth, A. (1994): Kampf um Anerkennung, Frankfurt a. M., Suhrkamp.

HEIDEGGER, M. (1986): Sein und Zeit, Tübingen, Niemeyer.

KAMBARTEL, F. (1991): «Versuch über das Verstehen», en McGuINEs, B. (ed.), Der Löwe spricht... und wir können ihn nicht verstehen, Frankfurt a. M., Suhrkamp, pp. 121-138.

KetTNER, M.: «Gute Gründe. Thesen zur diskursiven Vernunft», en APEL, K.-O. (ed.), Die eine Vernunft und die vielen Rationalitäten, Frankfurt a. M, Suhrkamp.

LYOTARD, J.-F. (1979): La condition postmoderne; rapport sur le savoir, París, Minuit.

- (1983): Le Différend, París, Minuit.

- (1986): «Grundlagenkrise», Neue Hefte für Philosophie, núm. 26, pp. 1-33.

Merleau-Ponty, M. (1945): Phénoménologie de la perception, París, Gallimard.

- (1960): Signes, París, Gallimard.

- (1962): «Un inédit de Maurice Merleau-Ponty", Revue de Métaphysique et de Morale, núm. 4, pp. 401-409.

- (1964): Le visible et l'invisible, París, Gallimard.

NABERT, J. (1924): L'expérience intérieure de la liberté, París, PUF.

- (1957): «La philosophie réflexive», en Encyclopédie française, XIX 19.04-14/10.06-3.

- (1971): Éléments por une éthique, París, Aubier (orig. 1943).

Peirce, Ch. S.: Collected Papers, Hartshorne, C., Weiss, P., Burks, A. (eds.), Cambridge, Harvard University Press, MA 1931-1958.

Ricoevr, P. (1985): Temps et récit, París, Seuil.

- (1990): Soi-Même comme un autre, París, Seuil.

RoRTY, R. (1979): Philosophy and the Mirror of Nature, Princeton University Press. 
- (1982): Consequences of Pragmatism, University of Minnesota.

- (1989): Contingency, irony and solidarity, Cambridge University Press.

- (1991): «Wittgenstein, Heidegger und die Hypostasierung der Sprache», en McGuines, B. (ed.), Der Löwe spricht...und wir können ihn nicht verstehen, Frankfurt a. M., Suhrkamp, pp. 69-93.

- (1994): «Sind Aussagen universelle Geltungsansprüche?», Deutsche Zeitschrift für Philosophie, 42, 6, pp. 975-988.

SÁEZ RuEDA, L. (1995): La reilustración filosófica de K.-O. Apel, Universidad de Granada.

- (1996a): «Für ein "tragisches" und "offenes" Konzept der Rationalität», Deutsche Zeitschrift für Philosophie, 44, 3, pp. 343-361.

- (1996b): «Por una diferencia no indiferente - a propósito de las críticas de Rorty y Lyotard a la nueva Ilustración alemana», ER, Revista de Filosofía, núm. 20, pp. 79-109.

- (1996c): «Segregación o domesticación de la experiencia prerreflexiva», Volubilis, núm. 4, pp. 35-53.

- (1998): «Das Unverfügbare und der Diskurs - Heideggers Vermächtnis im Streit von Moderne und Postmoderne-», Wiener Jahrbuch für Philosophie, núm. 11, pp. 191-214.

SCHNÄDELBACH, H. (1982): «Bemerkungen über Rationalität und Sprache», en Kunlmann, W., y BöHLeR, D. (ed.), Kommunikation und Reflexion, Frankfurt a. M., Suhrkamp.

Tugendhat, E. (1979), Selbstbewußtsein und Selbstbestimmung, Frankfurt a. M., Suhrkamp.

WALDENFELS, B. (1985): In den Netzen der Lebenswelt, Frankfurt a. M., Suhrkamp. - (1995): Deutchs-Französische Gedankengänge, Frankfurt a. M., Suhrkamp. Wellmer, A. (1986): Ethik und Dialog, Frankfurt, Suhrkamp.
1 Cf. K.-O. Apel, «Der transzendentalhermeneutische Begriff der Sprache», en 1973, II, pp. 330-358. 2 L. Sáez Rueda, «Für ein "tragisches" und "of fenes" Konzept der Rationalität», Deutsche Zeitschrift für Philosophie, 44, 1996, 3, pp. 343-361.

${ }^{3}$ Cf. Ch. S. Peirce, Collected Papers, 5, pp. 400 y ss. Cf. K.O. Apel, 1987, pp. 121 y.ss.

${ }_{4}$ Pues - como advierte explícitamente Apel - una Idea Regulativa está referida a principios normativos que son vinculantes para la acción, en la medida en que demandan la realización aproximativa de un ideal, el cual, sin embargo, no podrá alcanzar jamás plena realización en la facticidad: «[normative Prinzipien], die für das Handeln im Sinne einer Verpflichtung und Anleituing zur langfristigen, approximativen Realisierung eines Ideals verbindlich sind, die aber zugleich die Einsicht zum Ausdruck bringen, daß nichts in der Zeit Erfahrbares jemals dem Ideal völlig entsprechen kann». K.-O. Apel, 1988, p. 204.

5 Cf. R. Rorty, 1982, cap. 9, parte primera

${ }^{6}$ Cf. R. Rorty, 1989 , caps. 5 y 6.

${ }^{7}$ Cf. R. Rorty, 1989, cap. 4.
${ }^{8}$ Cf. R. Rorty, 1994, p. 982.

${ }^{9}$ Cf. J. Habermas, 1991a, pp. 127-157.

10 «El pensamiento humano - dice Apel-, si pretende ser radical, debe hacer uso de esta posibilidad constitutiva para él [la de la "posición excéntrica"], y que consiste en el distanciamiento con respecto al mundo y en el autodistanciamientos (1973, II, trad. cast., p. 374), aunque, simultáneamente, «una pura conciencia del objeto, tomada por sí sola, no puede extraer del mundo ningún sentido. Para lograr una constitución del sentido, la conciencia - esencialmente "excéntrica"- debe comprometerse céntricamente, corporalmente, aquí y ahora» (ibid., p. 93).

"1 K.-O. Apel, 1988, p. 101

${ }_{12}$ En el contexto de una filosofía trascendental transformada, el consenso ideal correspondería, según Apel, al «punto supremo» de la teoría kantiana (la «síntesis trascendental de la apercepción»), es decir, debería ser entendido como la «síntesis trascendenta de la interpretación lingüísticamente mediada" (trans zendentale Synthesis der sprachvermittelten Interpretation) y como la unidad del entendimiento sobre algo 
en una comunidad de comunicación (Einheit der Verständigung über etwas in einer Kommunikationsgemeinschaft). Cf. K.-O. Apel, 1973, I, p. 354. Mediante una ampliación de la semiótica de Peirce supone Apel, además, que la comunidad ideal sirve de idea regulativa respecto a un proceso histórico infinito en el que tiene lugar una progresiva «explicación del sentido» (Erklärung des Sinn), de forma que la Idea Regulativa de un discurso libre representa al mismo tiempo la idea de una síntesis de la validación de las interpretaciones. Cf. K.-O. Apel, 1987, \$ 4.1.

${ }_{13}$ Reconoce Habermas que las aspiraciones universalistas quedarían vacías o serían impotentes si a la anticipación de una comunidad ideal de comunicación no perteneciese también la conciencia de un hermanamiento en una forma de vida común («daß jede Universalisierungsforderung ohnmächtig bleiben müßte, wenn nicht auch noch aus der Angehörigkeit zu einer idealen Kommunikationsgemeinschaft ein Bewußtsein unkündbarer Solidarität, die Gewißheit der Verschwisterung in einem gemeinsamen Lebenszusammenhang entspringen würde»), a lo que añade que la justicia es impensable sin la idea de una reconciliación. Habermas, 1991b, p. 72. Podrían añadirse más ejemplos en esta línea. Se da el caso también de una coincidencia entre cumplimiento de las condiciones formales del ideal anticipado y el logro de una humanidad autónoma y emancipada. Como se sabe, Habermas comprende las fuerzas heterónomas que limitan la libertad como formas de un discurso distorsionado. Las condiciones de la autonomía vendrían a coincidir con las condiciones formales de un entendimiento racionalmente motivado, cuyo modelo reside en las condiciones formales de un diálogo sin coacciones. Tales condiciones representarian también las de una humanidad emancipada. Y no solamente esto, sino que incluso, en la medida en que la identidad, el Selbst, según Habermas, se forja en procesos de reconocimiento intersubjetivo, tales condiciones formales coinciden con las de la autorrealización. Tanto la autodeterminación como la autorrealización presuponen -dice Habermas- el reconocimiento de una comunidad de comunicación; el sí mismo no es pensable sin semejante reconocimiento («die Anerkennung einer unbergrenzten Kommunikationsgemeinschaft (...). Entsprechend kann sich je meine Identität, nämlich mein Selbstverständnis als eines autonom handelden und individuirten Wessen, nur stabilisieren, wenn ich als eine solche und als diese Person Anerkennung finde»). Habermas, 1992, p. 233.

${ }^{14} \mathrm{He}$ intentado justificar esta tesis ampliamente en L, Sáez Rueda, 1995, parte tercera.

15 Cf. A. Wellmer, 1986, pp. 62 y ss.

16 M. Kettner, 1996, p. 426, nota 12

17 Cf. J. Habermas, 1981, I, pp. 102 y ss., 148 y ss., 262 y ss.; II, pp. 173 y ss.

${ }^{18}$ Cf. M. Merleau-Ponty, 1945, pp. 119-160 y 181-183. Valga el ejemplo de que conoce perfectamente en qué consiste un partido político y qué opcio- nes se le presentan, pero no puede mantener opiniones políticas.

${ }_{19}$ W. Blankeniburg, 1971.

${ }^{20}$ Este sustrato de horizontes no refiere meramente a los intereses concretos y explicitables, sino a las for mas inobjetivables de organizar los nexos de sentido de la experiencia, sin cuya base las intenciones concretas y los intereses formulables carecerían de «significatividad" para el sujeto.

${ }^{21}$ M. Heidegger, Sein und Zeit, $\S 61$. P. Ricoeur, 1990 , pp. $118-120$ y $341-349$.

${ }^{22}$ Cf. M. Merleau-Ponty, 1945, parte tercera, capítulo 3. Sobre ello, véase el excelente trabajo de D. Blanco Fernández, 1994.

${ }^{23}$ Cf. J. Habermas, 1992, parte tercera, capítulo 8.

24 J. Habermas, 1992, pp. 32-33.

${ }^{25}$ Ver P. Ricoeur, 1985, tercer volumen; 1990, estudios quinto y sexto

${ }^{26}$ Cf. A. Honneth, 1994, pp. 212-227 y 256-288. Sus observaciones en torno a la idea de que la ausencia de reconocimiento, la experiencia de Mißachtung, impide una autorrelación libre y productiva, es decir, la integridad y el despliegue de las propias expectativas de autorrealización me parecen difícilmente rebatibles.

${ }^{27}$ La dimensión excéntrica presupone, como hemos dicho, la dimensión céntrica, pero, al mismo tiempo, una identidad narrativa, precisamente por estar articulada por una comprensión centrada, es demasiado próxima como para que pueda ser llamada «mía». Para aprehender el valor de la diferencia que la constituye de su irrepetibilidad, el sujeto ha de poder adoptar una perspectiva excéntrica.

${ }_{28} \mathrm{Cf}$. E. Tugendhat, 1979, Vorlessung 10.

${ }^{29}$ Ese esfuerzo por ser que reclama de nosotros aventurarnos en proyectos y en apuestas cuyo horizonte rebasa toda posible justificabilidad argumentativa $y$ cuyo reconocimiento posible no puede dejar de ser anhelado, pero tampoco erigido en causa primera o en fin último de la acción.

${ }^{30}$ Cf. K.-O. Apel, 1989.

${ }^{31}$ K.-O. Apel, 1973, II, p. 400.

32 J. Habermas, 1986.

${ }^{33}$ Cf. R. Rorty, 1991, pp. 91 y ss.

a 24 Ver K.-O. Apel, 1976.

35 K.-O. Apel, 1987, \$ 4.1.

${ }^{36}$ Ver M. Merleau-Ponty, 1964, el capítulo dedicado a «interrogación e intuición».

${ }^{37}$ Cf. B. Waldenfels, 1995 , pp. 435 y ss.

${ }^{38} \mathrm{M}$. Merleau-Ponty, «Le philosophe et son ombre», 1960, p. 210.

${ }^{39}$ Cf. Waldenfels, 1985 , pp. 115 y ss.

${ }^{40}$ Cf. M. Merleau-Ponty, 1962, pp. 406-407, y 1960 pp. $214-228$.

${ }^{41}$ Por ejemplo, J.-F. Lyotard, 1983, \& 126, y 1986 ${ }^{42}$ Cf. Lyotard, 1983, pp. 9 y ss., y $\$ \$ 40,91,183$, 188.

${ }^{43}$ Esto no significa, como piensa M. Frank, que Lyotard incurra en un misticismo según el cual «der Sprache Spricht», sino que las intenciones de sentido de los agentes ingresan en un movimiento lingüístico 
cuya productividad rebasa las intenciones. Cf. $M$. Frank, 1988, pp. 49-56. En una línea compatible con la que he señalado ha mostrado Kambartel que, puesto que la participación en juegos lingüísticos implica un uso de reglas que es indeterminable a priori, el significado de nuestras propias palabras, que se constituye en el magma de ese juego, es para nosotros unverfügbar. Cf. F. Kambartel, 1991, pp. 126 y ss.

44 He abordado este tema más extensamente en $L$. Sáez Rueda, 1996 b y 1997.

${ }^{45}$ Debo a las sugerencias del Profesor D. Blanco Fernández el haberme hecho cargo de las profundas reflexiones de J. Nabert.

${ }^{46}$ Cf. J. Nabert, 1971, pp. 19-47, y cap. VII («le devoir et l'existence»).

47 Ver, en general, J. Nabert, 1957

48 Cf. J. Nabert, 1924, pp. 123-155.

49 Cf. J. Nabert, 1971, pp. 82-87.

${ }^{50}$ Esto se pone de manifiesto con claridad, por ejemplo, en la tesis apeliana según la cual la pretensión fundamental inscrita en la dimensión performativa del habla es la pretensión de sentido válido intersubjetivamente $y$, por así decirlo, intemporal («Anspruch auf intersubjektiv gültigen und sozusagen zeitlosen Sinn»), pretensión que implica, según Apel, el postulado de una posible explicación del sentido: «muß man mit einem potentiell unendlichen Proze $\beta$ der Zeicheninterpretation (Peirce) als Explikation von Sinn gerechnet werden». K.-O. Apel, 1996, p. 22.

${ }^{51} \mathrm{He}$ abordado este tema con mayor extensión en L. Sáez Rueda, 1996a.

${ }^{52}$ Cf. H. Schnädelbach, 1982 , pp. $354-360$

$53 \mathrm{Cf}$. A. Wellmer, 1986, pp. 220 y ss.

${ }_{54}$ J. Habermas, 1986, trad. cast., p. 37. ss Ver, por ejemplo, Jocelyn Benoist (1994): Autour de Husserl. L'Ego et la Raison, París, Vrin; Jean-François Courtine (1990): Heidegger et la Phénoménologie, París, Vrin; Natalie Depraz (1994): «Temporalité et affection dans les manuscrits tardifs sur la temporalité (1929-1935) de Husserl», en Alter. Revue de Phénoménologie, núm. 2, pp. 63-86; Didier Franck (1981): Chair et corps. Sur la Phénoménologie de Husserl, París, Minuit; M. Haar (1984): «La chair et la constitution temporelle», en Jean Luc Marion y G. Planty (1984 eds.): pp. 125-156; Michel Henry (1990): Phénoméno logie materielle, París, PUF; Dominique Janicaud (1995, ed.): L'intentionalité en question, París, Vrin.

56 Esp. significativo, D. Blanco Fernández, 1998. Salvando las distancias, como las que afectan a la idea de «oposición trágica» y al concepto de «idea regulativa», la concepción de la acción racional como una instancia tensional, que constituye el punto de partida del presente escrito, es coincidente con el posicionamiento básico de Domingo Blanco en sus trabajos.

${ }^{57}$ Cf. D. Goleman, 1996. Goleman destaca, por ejemplo, estudios en psicología del desarrollo en los cuales se detecta que los niños sienten angustia y reaccionan ante disturbios en el entorno incluso antes de ser capaces de comprender que existen independientemente de otras personas y de hacerse cargo conscientemente de la posibilidad del sufrimiento del otro, pp. 38-45.

58 Ver A. R. Damasio, Descates' error. Emotion, Reason, and the Human Brain, Nueva York, 1994.

59 Ver P. Ricoeur, 1990, cap. 10, § III.1.

${ }^{60}$ Merleau-Ponty apunta en esa línea. Cf. 1962 También M. Haar, 1994, pp. 244 y ss. 